\title{
Validity of the thin mask approximation in extreme ultraviolet mask roughness simulations
}

\author{
Patrick P. Naulleau and Simi A. George \\ Center for X-Ray Optics, Lawrence Berkeley National Laboratory, Berkeley, CA 94720
}

\begin{abstract}
In the case of extreme ultraviolet (EUV) lithography, modeling has shown that reflector phase roughness on the lithographic mask is a significant concern due to the image plan speckle it causes and the resulting line-edge roughness on imaged features. Modeling results have recently been used to determine the requirements for future production worthy masks yielding the extremely stringent specification of $50 \mathrm{pm}$ rms roughness. Owing to the scale of the problem in terms of memory requirements, past modeling results have all been based on the thin mask approximation. EUV masks, however, are inherently three dimensional in nature and thus the question arises as to the validity of the thin mask approximation. Here we directly compare image plane speckle calculation results using the fast two dimensional thin mask model to rigorous finite-difference time-domain results and find the two methods to be comparable.
\end{abstract}

Keyword: extreme ultraviolet, lithography, multilayer, mask, line-edge roughness 


\section{Introduction}

Line edge and width roughness (LER and LWR) remains one of the most daunting challenges facing the commercialization of extreme ultraviolet (EUV) lithography. Mask contributors, especially multilayer phase roughness have recently been shown to be significant contributors to LER [1-4]. Phase roughness at the mask couples to the intensity variations at the wafer through the concept of speckle [2,3] and thus LER. Since this effect has been experimentally verified [5], there has been significant interest in the implications it has on EUV mask phase roughness specifications and thus substrate roughness specifications. This question has been addressed in the past [6] using two dimensional (2D) scalar modeling with the thin mask approximation [4] yielding the stringent specification of $50 \mathrm{pm}$ replicated surface roughness [6] for 22-nm half-pitch device fabrication. Thin mask modeling was used due to the significant memory and speed burdens that would be incurred with full three dimensional finite-difference time-domain (FDTD) modeling. Being Bragg structures, however, EUV masks are certainly three dimensional (3D) in nature and thus the question naturally arises as to the validity of the simplified modeling. Here we answer this question through direct comparison of the simplified thin mask model to rigorous FDTD in the computation of image plane speckle from an EUV mask with surface (phase) roughness.

To give the reader an idea of the speed differences between the two methods we first consider the case of a $4 \times$ mask of $2 \mu \mathrm{m}$ width (mask dimensions) with only 10 bilayers, a lateral grid size of 10 $\mathrm{nm}$, longitudinal grid size of $0.5 \mathrm{~nm}$, numerical aperture of 0.32 , and partial coherence of 0.5 . Using a state-of-the-art quad-core machine, a through focus (21 points) series with full FDTD modeling takes approximately 12 hours to run as compared to approximately 30 seconds for 2D modeling. Moreover, attempting a lateral grid size of 1 -nm would require $200 \mathrm{G}$ bytes of memory which is not feasible on a typical desktop computer. In the 2D case, however, a 1-nm lateral grid size is readily achievable. 


\section{Modeling methodology}

The simplified model uses scalar aerial image computation software based on the partiallycoherent image formation equations [7]. Similar capabilities can also be obtained through the use of commercial modeling packages such as Prolith [8] and HyperLith [9]. In this model, the multilayer roughness is modeled as simple phase variations in the object plane. Accounting for reflection from the Bragg structure and assuming the structure to be intact throughout at least the top 10 layers of the multilayer stack, the phase variations are set to two times the multilayer height variations to be modeled.

For the rigorous 3D model we use the FDTD mask computation option in the Panoramic Technology EM-Suite software package. In this case, the mask is modeled as a full 3D Bragg structure with the roughness encoded into height variations of the various layers. Figure 1 shows a screen capture of the top 10 layers in the modeled surface where the white layers represent the molybdenum and the gray layers represent silicon. The black region at the top is vacuum. The thickness ratio of the two materials is set to optimize the reflectivity. As seen in Fig. 1, we assume the height variations to be perfectly replicated through the stack which represents the worst case in terms of induced phase roughness and makes the simulation directly comparable to the simplified model where we have taken the phase variations to be two times these height variations. We note that the aspect ratio in Fig. 1 is exaggerated to better visualize the replicated nature of the roughness. The grid spacing we use in the 3D case is $10 \mathrm{~nm}$ for $\mathrm{x}$ and $\mathrm{y}$ and 0.05 $\mathrm{nm}$ for $\mathrm{z}$. These values were determined empirically based on stability of the results as a function of decreasing grid size.

In the modeling we assume the roughness to have a correlation length of $125 \mathrm{~nm}$ on the mask and have an rms height variation of $230 \mathrm{pm}$ and we use the same statistical realization of 
the roughness for the two cases. The total mask used in the model is $2 \mu \mathrm{m} \times 2 \mu \mathrm{m}$.

\section{Comparison results}

We begin with the direct comparison of the two modeling methods assuming an angle of incidence of $0^{\circ}$ at various numerical apertures (NAs). Figure 2 shows good agreement in the computed through focus speckle contrast within the depth of focus of the optical system. The error increases with NA and peaks at approximately two times the depth of focus where we find a maximum error of about $10 \%$.

Next we consider the effect of angle of incidence (AOI) on the results. Heuristically one can argue that the AOI should have minimal impact as long as the lateral displacement of the ray traveling from one layer to the next is small relative to the correlation length of the roughness. When this is the case, the roughness a single ray sees at each surface remains correlated from surface to surface. With a bilayer thickness of approximately $7 \mathrm{~nm}$ and a critical layer number of 10 , we find the longitudinal ray displacement of interest to be $70 \mathrm{~nm}$. Given a correlation length of $125 \mathrm{~nm}$, we expect no effect on interlayer correlation until the AOI surpasses approximately $60^{\circ}$, which is well beyond any angles present on EUV masks. Taking an extreme case of 0.75 $\mathrm{NA}$ and an AOI of $9^{\circ}$, the maximum angle at the mask would only be $20^{\circ}$. Figure 3 compares the computed speckle contrast at angles of incidence of $0^{\circ}$ and $10^{\circ}$, respectively, using the 3D method as a function of NA. We see the results to be relatively insensitive to AOI for all considered NAs. This further indicates that the simplified model works even in the case of nonnegligible AOI.

Next we expand the 2D versus 3D modeling comparison to consider the case where absorber lines are also included in the simulation and the LER is directly measured instead of only comparing the image plane speckle. We consider the case of 22-nm half-pitch lines on a $4 \times$ 
mask with a 50-nm thick TaBN absorber. The optical system has a numerical aperture of 0.32 and is aberration free. The illumination coherence factor is set to 0.5. Figure 4 shows the direct comparison of the calculated results. On the positive side of focus (above the mask plane) very good agreement is found with an average error of $6 \%$ for defocus values of $20 \mathrm{~nm}$ and larger. At best focus we find that the 2D method under-predicts the LWR by $0.66 \mathrm{~nm}$ or $47 \%$. This underprediction is a result of an offset in best focus for the absorber lines compared to the multilayer surface that is accurately captured in the $3 \mathrm{D}$ model whereas it is forced to zero in the $2 \mathrm{D}$ case. As we focus into the mask, a larger discrepancy is seen with the 3D method predicting a larger depth of focus. This is likely due to the guiding effect of the thick absorber lines on the mask. This causes the $2 \mathrm{D}$ method to be a somewhat conservative predictor of mask specifications. To consider the importance of this effect we note that the mask roughness specifications are determined based on impact on depth of focus (DOF) [6]. The DOF difference between the two methods, assuming the DOF to be set by a 10\% LWR limit, is found to be approximately $20 \%$.

Finally, all modeling performed in this area to date has assumed that the roughness is conformally replicated through the multilayer stack, since that is the roughness that is thought to directly translate to phase in the reflected field. With the rigorous 3D modeling, however, we can explicitly consider the case where the roughness is uncorrelated from layer to layer. In this situation one would expect the phase deviations at the surface to be smaller than in the correlated case and we would further assume reflectivity variations to arise across the surface. We consider two different cases of uncorrelated layer roughness with the first case being conformal growth of a series of layers with uncorrelated thickness variation from layer to layer and the second case being fully uncorrelated interfaces throughout the multilayer stack. In both cases we consider a 0.32-NA system with no aberrations. 
In generating the multilayer for the first case (conformal growth with uncorreated random thickness layers), we assume the substrate to be perfectly smooth and the thickness variation of each layer to be $\operatorname{sqrt}(N)$ smaller than the total target top surface roughness of $230 \mathrm{pm}$, where $N$ is the total number of layers being modeled (Gaussian roughness is assumed). Note that although the thickness variation of all the layers is independent, the conformal growth still yields correlation from interface to interface as seen in Fig. 5. Figure 6 shows the through focus speckle results for a 40 bilayer multilayer demonstrating the same characteristic behavior expected from phase roughness, although the magnitude of the speckle is reduced. The peak speckle is seen to be reduced from approximately $17 \%$ to $14 \%$ and the best focus speckle contrast is reduced from $2 \%$ to $1 \%$.

Next we consider the case of fully uncorrelated interfaces from layer to layer. Although not physically realistic in terms of a typical film deposition process, this condition is instructive as it represents the opposite extreme condition of fully correlated interface roughness. In this case, we model the multilayer as a series of interfaces instead of layers where each interface has the full $230 \mathrm{pm}$ roughness. In the model, this is achieved by starting with a 230-pm rough layer on a perfectly smooth substrate and then planarizing the first layer with the material of the second layer and from that point adding a 230-pm rough layer of the second material while keeping the nominal thickness of the layer at the target. This process is then repeated for all 40 bilayers until the top where we end with a top surface interface to vacuum with roughness of 230 pm. For all layers except the very first layer, the thickness variation can be shown to be $\operatorname{sqrt}(2)$ times $230 \mathrm{pm}$ as a result of the uncorrelated nature of the upper and lower interface at each layer. Figure 7 shows a schematic of the modeled multilayer stack and Fig. 8 the resulting throughfocus speckle. In this case we observe dramatically lower speckle contrast, reinforcing the 
importance of the replicated nature of the interface roughness in the formation of random reflected phase and speckle.

\section{Summary}

Mask multilayer roughness has emerged as an important issue for EUV lithography. Study of this complex issue requires the use modeling techniques. Simplified 2D modeling has the significant advantage of greatly increased speed (approximately three orders of magnitude) and reduced memory requirements compared to rigorous $3 \mathrm{D}$ modeling. This is especially important in the case of LER modeling where relevant studies require large mask areas to be considered. The use of $2 \mathrm{D}$ techniques to solve a problem that is inherently $3 \mathrm{D}$ in nature, however, raises concerns. Here we have shown that for the purposes of mask-roughness induced speckle, simplified 2D modeling is indeed sufficient.

The authors thank Tom Pistor of Panoramic Technologies for valuable discussions and help with EM-Suite. This work was supported in part by SEMATECH through the U.S. Department of Energy under Contract No. DE-AC02-05CH11231 and carried out at Lawrence Berkeley National Laboratory.

\section{References}

1. P. Naulleau and G. Gallatin, "The line-edge roughness transfer function and its application to determining mask effects in EUV resist characterization,” Appl. Opt. 42, 3390-3397 (2003).

2. N. Beaudry, T. Milster, "Effects of mask roughness and condenser scattering in EUVL systems," Proc. SPIE. 3676, 653-662 (1999).

3. P. Naulleau, "The relevance of mask-roughness-induced printed line-edge roughness in recent and future EUV lithography tests," Appl. Opt. 43, 4025-4032 (2004).

4. P. Naulleau, D. Niakoula, G. Zhang, "System-level line-edge roughness limits in extreme ultraviolet lithography,” J. Vac. Sci. \& Technol. B 26, 1289-1293 (2008). 
5. P. Naulleau, "Correlation method for the measure of mask-induced line-edge roughness in extreme ultraviolet lithography,” Appl. Opt. 48, 3302-3307 (2009).

6. P. Naulleau and S. George, "Implications of image plane line-edge roughness requirements on extreme ultraviolet mask specifications," Proc. SPIE 7379, 73790O-73790O-11 (2009).

7. J. W. Goodman, Statistical Optics, John Wiley and Sons, New York, 1985, Chap. 7, 286360.

8. Prolith is a registered trademark of KLA-Tencor Corporation, 160 Rio Robles, San Jose, California 95134.

9. EM-Suite is a registered trademark of Panoramic Technologies, www.panoramictech.com. 


\section{List of Figures}

Fig. 1. HyperLith screen capture of the top 10 layers in the modeled surface where the dark layers represent the molybdenum and the light layers silicon.

Fig. 2. Direct comparison of two and three dimensional modeling methods for the computation of image plane speckle assuming an angle of incidence of $0^{\circ}$ at various numerical apertures.

Fig. 3. Computed speckle contrast using the three dimensional method as a function of numerical aperture and angle of incidence. Results are relatively insensitive to angle of incidence for all considered numerical apertures.

Fig. 4. Direct comparison of two and three dimensional modeling methods for the computation of image plane LER.

Fig. 5. Image of multilayer stack generated using uncorrelated layer thickness model. Aspect ratio is greatly exaggerated for visualization purposes.

Fig. 6. Speckle through focus for uncorrelated layer thickness roughness using 3D modeling.

Fig. 7. Image of multilayer stack generated using Uncorrelated interface roughness model. Aspect ratio is greatly exaggerated for visualization purposes.

Fig. 8. Speckle through focus for uncorrelated interface roughness using 3D modeling. 


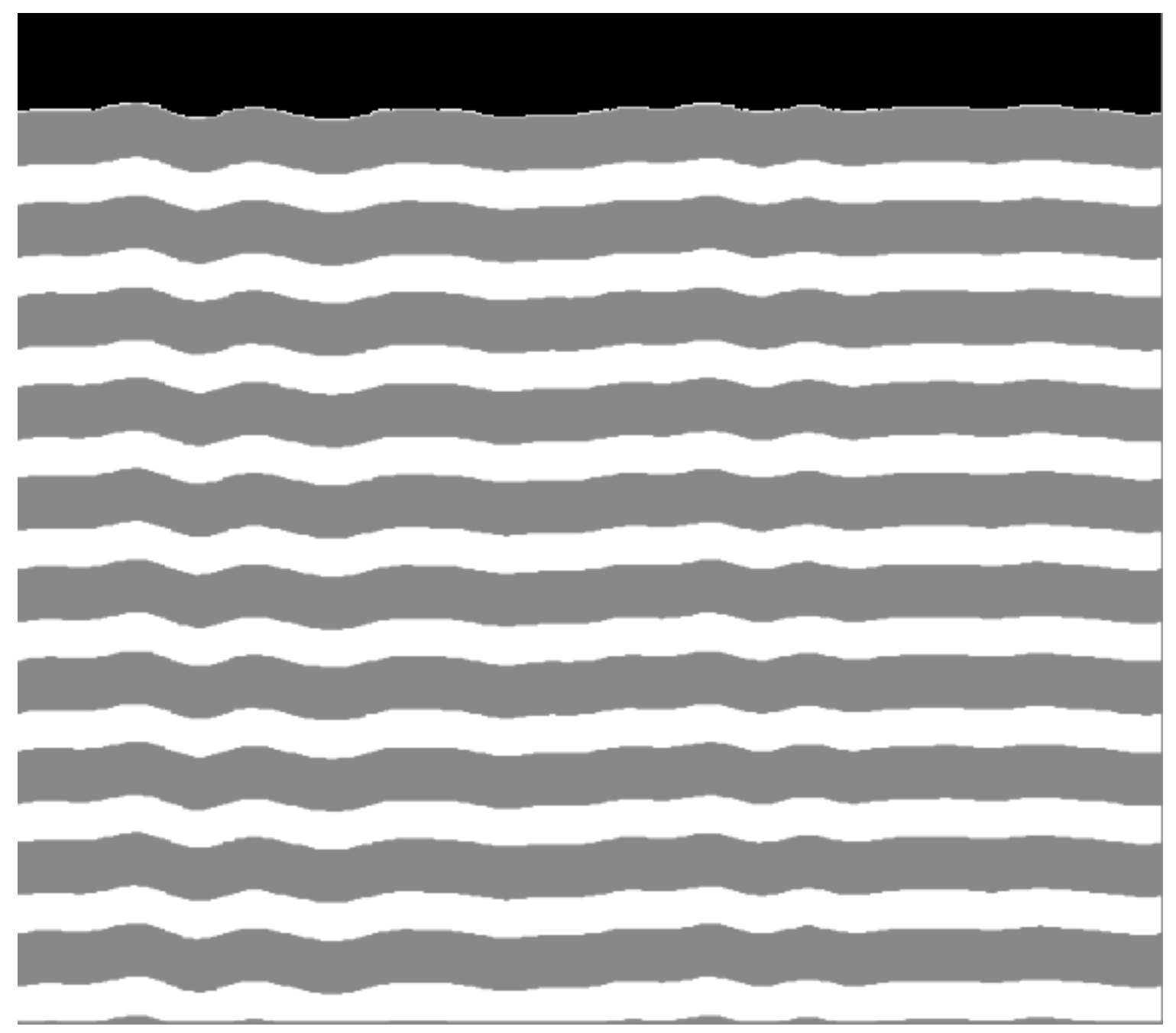

Fig. 1. HyperLith screen capture of the top 10 layers in the modeled surface where the dark layers represent the molybdenum and the light layers silicon. 


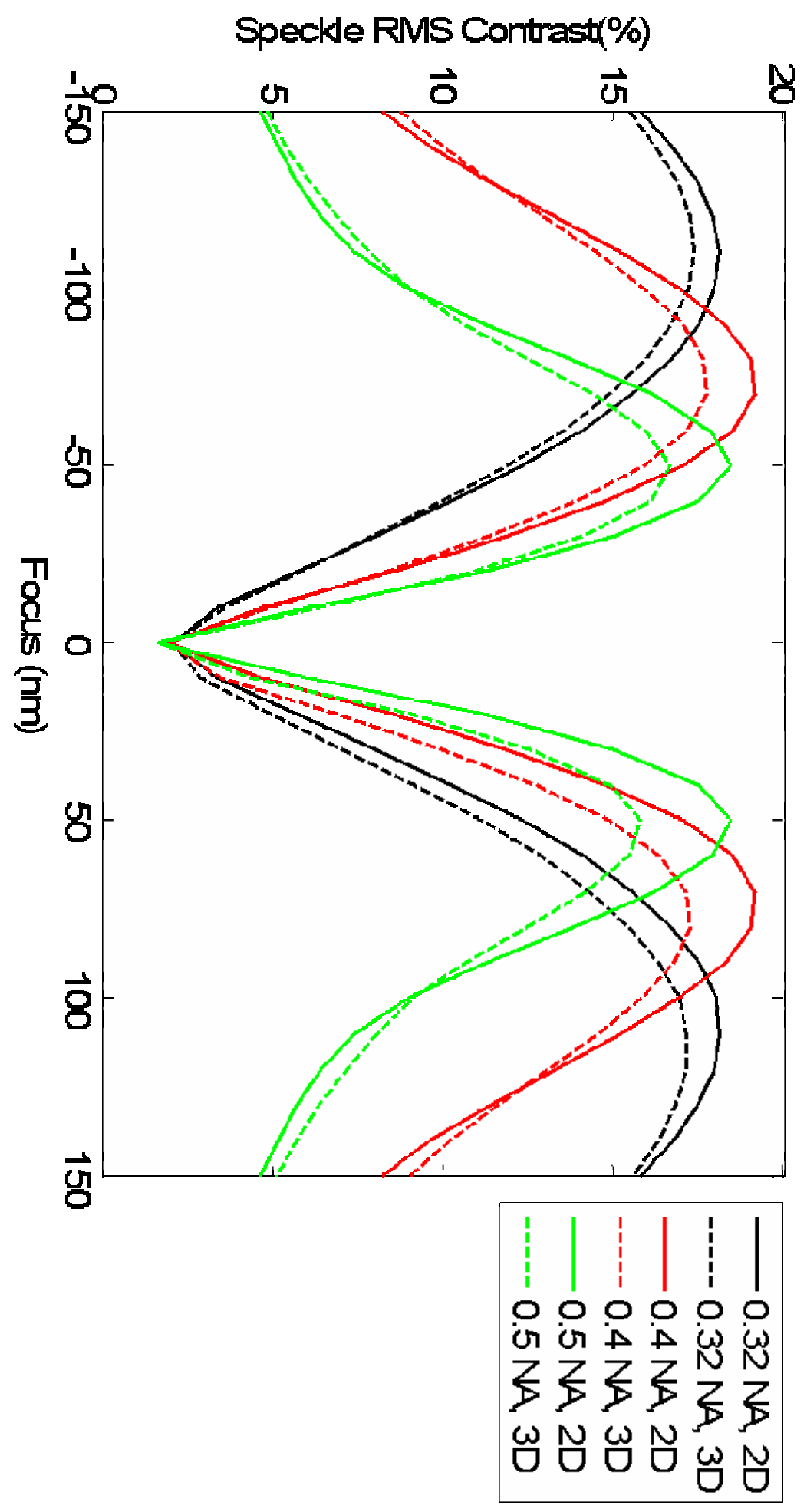

Fig. 2. Direct comparison of two and three dimensional modeling methods for the computation of image plane speckle assuming an angle of incidence of $0^{\circ}$ at various numerical apertures. 


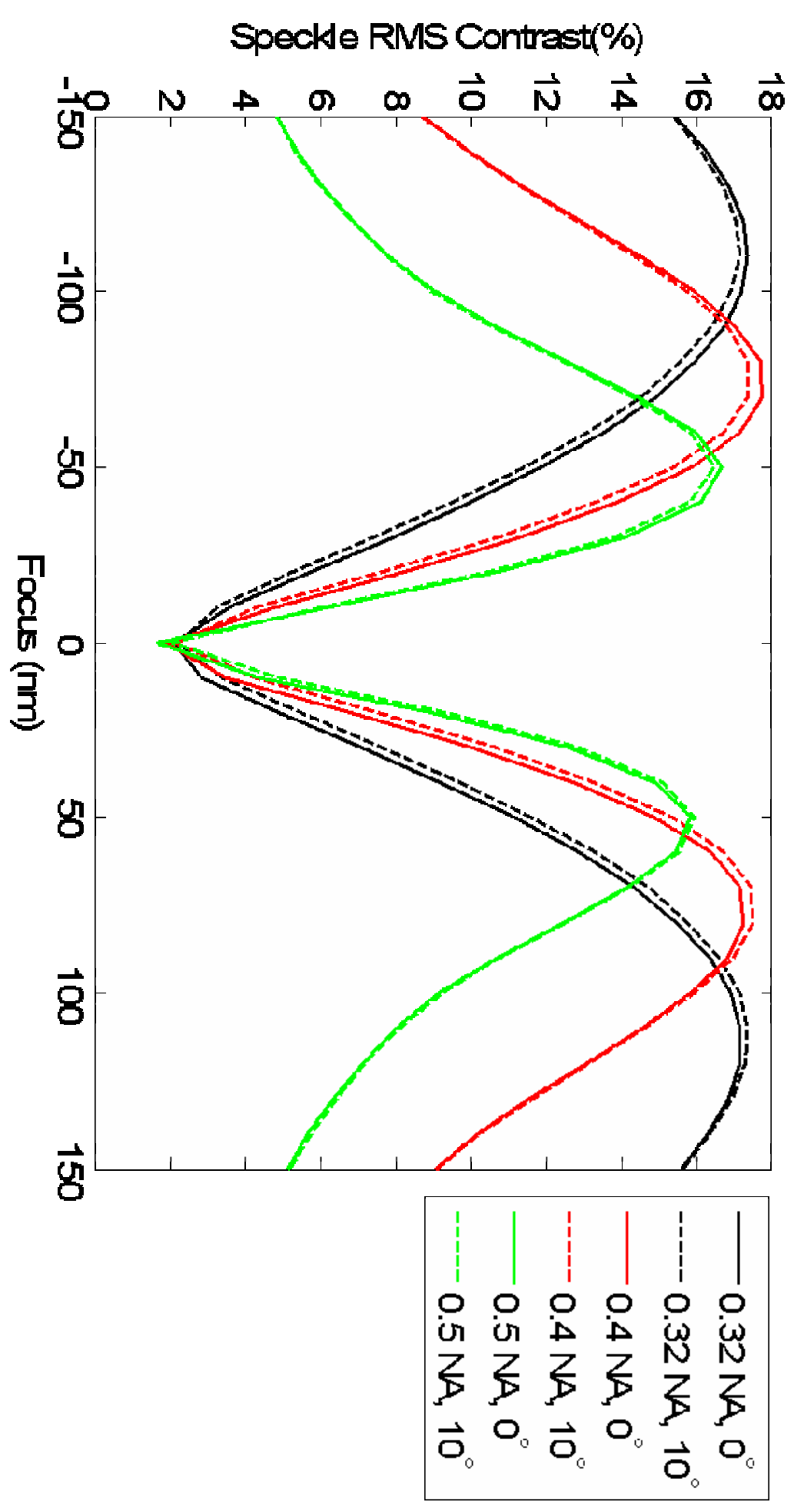

Fig. 3. Computed speckle contrast using the three dimensional method as a function of numerical aperture and angle of incidence. Results are relatively insensitive to angle of incidence for all considered numerical apertures. 


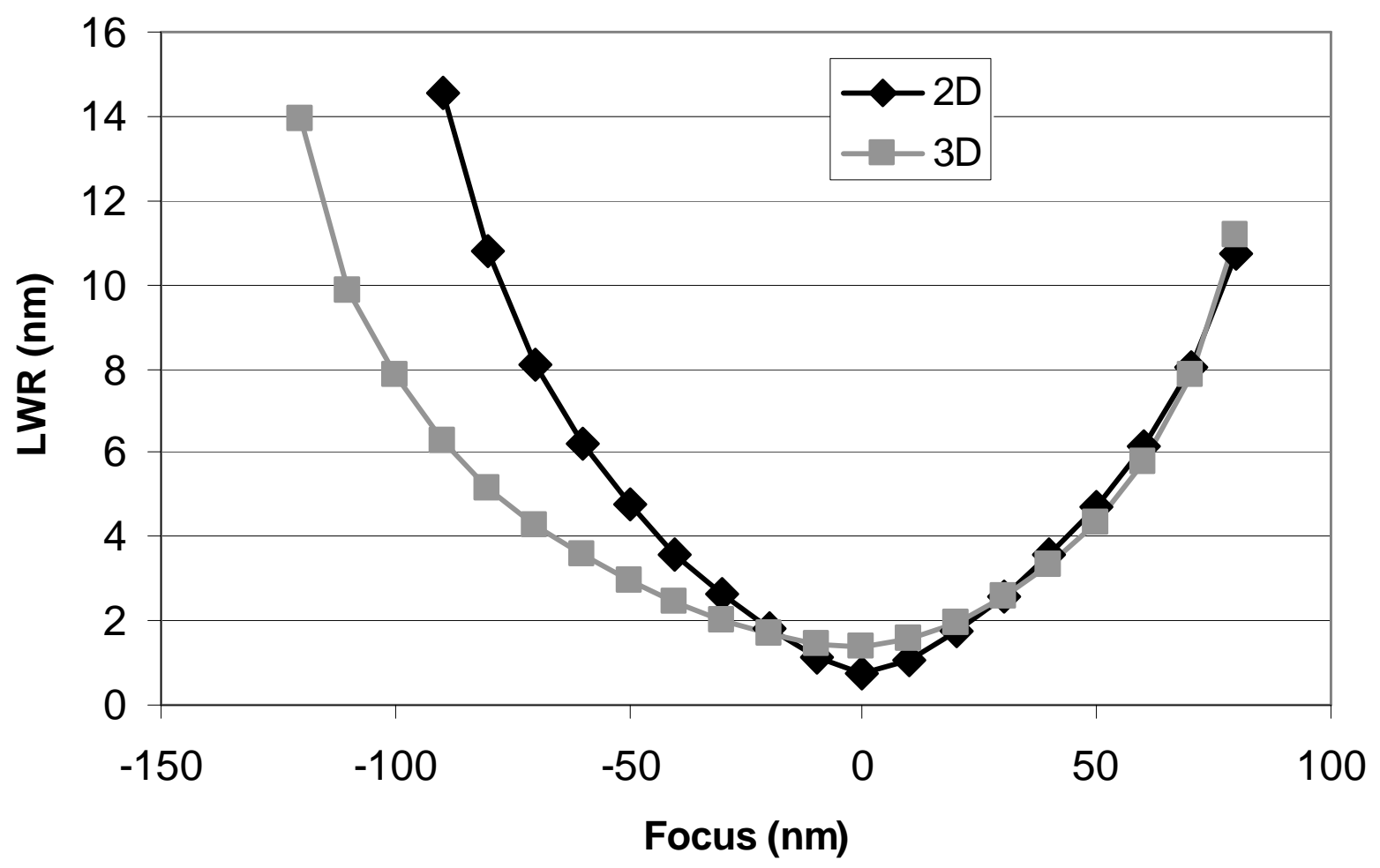

Fig. 4. Direct comparison of two and three dimensional modeling methods for the computation of image plane LER. 


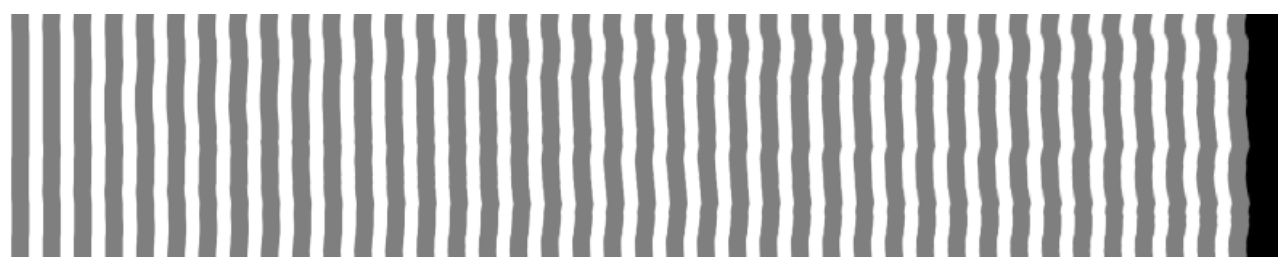

Fig. 5. Image of multilayer stack generated using uncorrelated layer thickness model. Aspect ratio is greatly exaggerated for visualization purposes. 


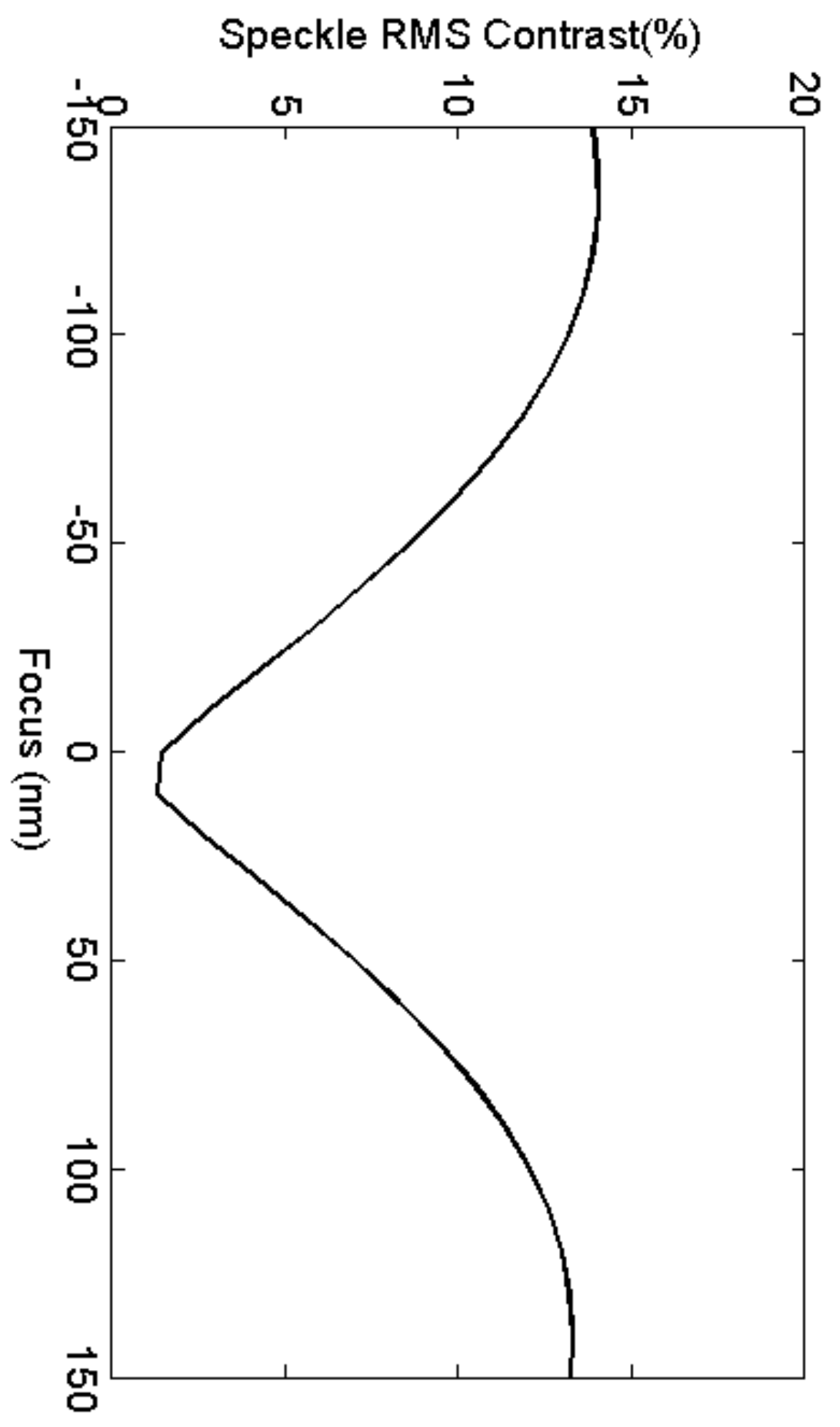

Fig. 6. Speckle through focus for uncorrelated layer thickness roughness using 3D modeling. 


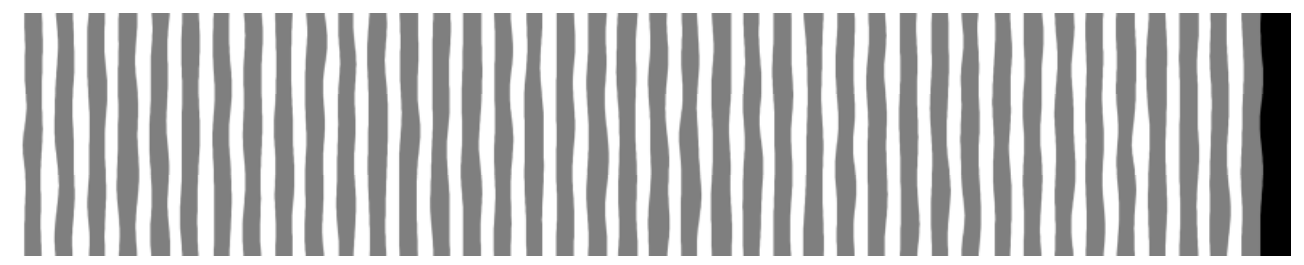

Fig. 7. Image of multilayer stack generated using Uncorrelated interface roughness model. Aspect ratio is greatly exaggerated for visualization purposes. 


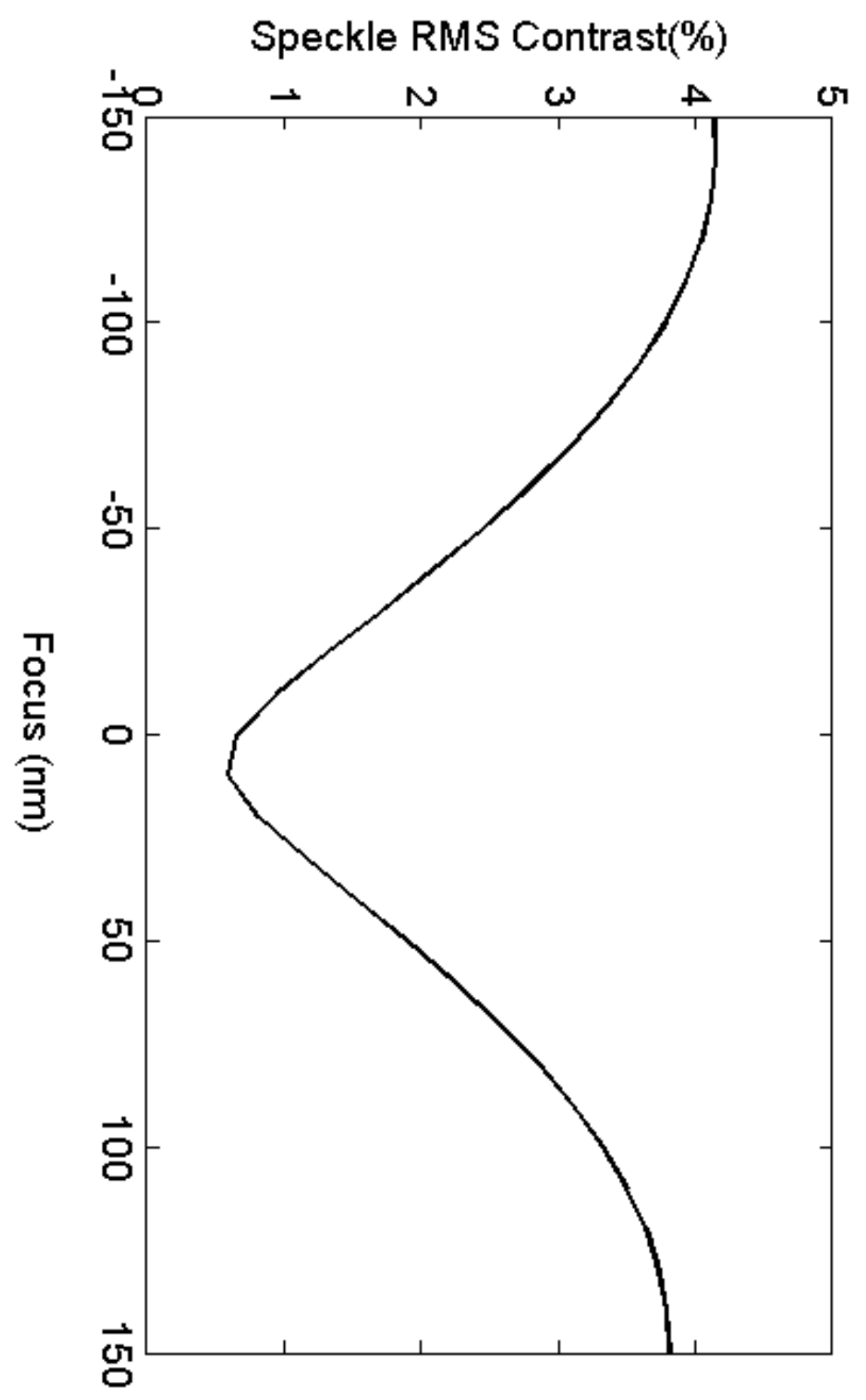

Fig. 8. Speckle through focus for uncorrelated interface roughness using 3D modeling. 


\section{DISCLAIMER}

This document was prepared as an account of work sponsored by the United States Government. While this document is believed to contain correct information, neither the United States Government nor any agency thereof, nor the Regents of the University of California, nor any of their employees, makes any warranty, express or implied, or assumes any legal responsibility for the accuracy, completeness, or usefulness of any information, apparatus, product, or process disclosed, or represents that its use would not infringe privately owned rights. Reference herein to any specific commercial product, process, or service by its trade name, trademark, manufacturer, or otherwise, does not necessarily constitute or imply its endorsement, recommendation, or favoring by the United States Government or any agency thereof, or the Regents of the University of California. The views and opinions of authors expressed herein do not necessarily state or reflect those of the United States Government or any agency thereof or the Regents of the University of California. 